\title{
Seeing the Signs of Workarounds: A Mixed-Methods Approach to the Detection of Nurses' Process Deviations
}

\author{
Iris Beerepoot \\ Utrecht University \\ i.m.berepoot@uu.nl
}

\author{
Xixi Lu \\ Utrecht University \\ x.lu@uu.nl
}

\author{
Inge van de Weerd \\ Utrecht University \\ i.vandeweerd@uu.nl
}

\author{
Hajo A. Reijers \\ Utrecht University \\ h.a.reijers@uu.nl
}

\begin{abstract}
Workarounds are intentional deviations from prescribed processes. They are most commonly studied in healthcare settings, where nurses are known for frequently deviating from the intended way of using health information systems. However, workarounds in healthcare have only been studied using qualitative methods, such as observations and interviews. We conduct a case study in a Dutch hospital and use a mixed-methods approach that draws not only on interviews and observations, but also on process mining, to detect and analyse eight workarounds that occur in a clinical care process. We contribute to theory by demonstrating that it is possible to use data to determine the occurrence of a rich variety of workarounds found using qualitative methods. Practically, this implies that workarounds that are identified qualitatively can be further analysed and monitored using quantitative methods. Once identified, workarounds also provide an attractive starting point for organisational learning and improvement.
\end{abstract}

\section{Introduction}

As healthcare professionals are frequently confronted with unpredictable situations, it happens that they deviate from procedure. So-called workarounds are defined as intentional deviations from prescribed practices $[2,8]$. They are often studied in relation to how prescribed practices are supported by information systems, and how these systems are used differently in practice [11]. Although workarounds can be regarded as harmful noncompliance to carefully designed procedures, there is another side to that coin [2]. Workarounds provide information systems users flexibility in dealing with unpredictable circumstances [21]. The COVID-19 crisis attests how important such flexibility actually is. Workarounds can also be seen as sources of valuable knowledge on what blockages users perceive in their daily work [8]. Studying them enables organisations to analyse organisational performance and improve processes [21].

To date, there is a large body of knowledge on workarounds that are identified with qualitative methods, particularly in the healthcare sector $[5,9$, 14, 29]. However, qualitative methods are labourintensive and it is uncertain whether they are effective to determine whether users reveal all their workaround behaviour [6]. Additionally, qualitative methods make it difficult to collect information on the frequency of workarounds and their evolution over time [12]. Recently, attempts have been made to detect workarounds quantitatively using process mining $[19,27]$. Process mining techniques use socalled event logs, extracted from an IT system, to perform process analyses on those data. Early studies have demonstrated that some types of workarounds are detectable with process mining. Utilising qualitative as well as quantitative approaches can enable the preliminary qualitative identification of workarounds, which can then be further analysed and monitored by studying workaround behaviour in data. Additionally, using quantitative methods, new types of workarounds may be found in addition to the ones established using qualitative methods. Therefore, there is a clear need to evaluate the suitability of a mixed-methods approach to detecting and analysing workarounds $[8,10]$.

It is an open question whether quantitative workaround detection - in addition to qualitative detection - is possible in a healthcare setting. The few quantitative workaround studies to date were conducted in sectors that are very different from healthcare. Healthcare processes are particularly complex, involve many different actors, and are characterised by high uncertainty $[25,28]$. Therefore, it is reasonable to expect different types of workarounds in the healthcare processes than seen in other domains. Additionally, existing studies focus 
predominantly on control-flow workarounds, i.e. situations where users deviate from the prescribed order of activities. This is arguably a rather narrow perspective, since many other perspectives on work processes exist.

With this study, we aim to enable the detection of workarounds specifically in healthcare processes. Furthermore, we purposefully take a broad perspective on processes by looking beyond the control-flow perspective, e.g., by also considering timing aspects. We carried out six case studies, collecting data from a number of healthcare professionals and analysing large sets of operational event data. For this analysis we use process mining techniques next to observations and interviews, which is a novel approach. Our main contribution is that we demonstrate a mixed-methods approach to the detection of a set of very diverse workarounds. We illustrate how certain characteristics in the data signal the existence of workarounds, which can then be quantitatively processed. In addition, we suggest how healthcare organisations can keep such workarounds under control and use these as a starting point for quality improvement. This specifically answers the call formulated in [21].

The paper is structured as follows. We start by synthesising the existing body of knowledge on workarounds and their detection using qualitative and quantitative methods in Section 2. Subsequently, in Sections 3 and 4, we describe our research approach and present the results of our case study, respectively. We discuss the implications of the results to theory and practice in Section 5 before concluding this paper with Section 6.

\section{Theoretical Background}

\subsection{Definition and Detection of Workarounds}

In the Information Systems discipline, there is an ongoing debate on how workarounds need to be defined. In most studies, they have four characteristics ascribed to them [10]. The first is that there is a certain designed path, the norm on how work should be done. The second is that users perceive some kind of block in the way the ideal path is meant to be followed. Users come up with a workaround that is aimed at achieving the same, overall goal as the normative path, which is the third characteristic. Fourth and last, the workaround is intentional, i.e. the deviation is not a mistake or an instance of fraud or sabotage.

Apart from a few exceptions, workarounds have only been identified using qualitative data collection methods, such as interviews, observations, and document analysis [10]. To the best of our knowledge, there are only four works that use quantitative methods to study workarounds. Two studies by Laumer et al. [15] and Van de Weerd et al. [26] are similar in that the interviews are paired with a survey to enrich the information collected on workarounds. A third study by Weinzierl et al. [27] draws on process mining and machine learning techniques to detect workarounds in open datasets with artificially added deviations to them. In a fourth study by Outmazgin and Soffer [19], a real-life dataset was used to detect workarounds in a purchasing and intake processes. The authors distinguished six generic workaround types, of which four were considered detectable. The studies by Weinzierl et al. and Outmazgin and Soffer demonstrate that process mining techniques have the potential to detect workarounds using quantitative techniques, i.e. by the analysis of data. However, they also show that not all workarounds are detectable using process mining, and that workaround information obtained qualitatively is necessary to get a complete picture of deviant behaviour.

The open question that concerns us in this work is how qualitative detection methods and process mining can be combined to detect and analyse workarounds in healthcare. This is of interest since healthcare is the domain that has been the focus of workarounds research, while it is also known for its complex processes involving many different actors. The question is relevant because the use of a mixedmethods approach to study workarounds in healthcare can enable a more complete identification of workarounds, and possibly provide new quantitative insights and theories [10].

\subsection{Multi-Perspective Conformance Checking}

In order to explore the quantitative detection of workarounds in healthcare, we draw on the field of compliance checking (cf. Outmazgin and Soffer [19]). Workarounds can be viewed as a form of intentional incompliance. Specifically, taking into account the characteristics of workarounds as mentioned in the previous section, workarounds are instances of intentional noncompliance where the goal remains the same as when following the designed path.

In the context of process mining, compliance is commonly analysed using conformance checking techniques. Along with discovery and enhancement, conformance has always been one of the main types of process mining [1]. For all three types of process 
mining, an event $\log$ is necessary. An event log consists of a number of events that usually contain at least the following information for each event: the activity that was executed (e.g. recording a patient's heart rate), the case it refers to (e.g. a specific patient or patient admission, also often referred to as process instance), and the timestamp (the date and time the event was executed). The event log can also contain information on the resources that execute the activities or include additional data attributes. When using process mining for conformance, an event log is checked against a set of rules or model that indicates how the process should run. One of the classic examples of this is checking whether the foureyes principle has been enforced. In the context of healthcare this could relate to two nurses checking medication to ensure that the right medication is given to the right patient.

Even though conformance is considered essential in order to improve processes, it has not received nearly as much attention as discovery [18]. Within conformance checking research, there is a strong emphasis on the control-flow perspective of a process, which refers to the order of activities in the process. Other perspectives such as the data, resource, and time perspective are often considered 'second-class citizens' [17]. The data perspective relates to the variables that are associated with cases and that may be modified during the execution of activities. In the context of conformance, taking a data perspective involves analysing the conditions behind the execution of paths within the process. Taking the process of recording a patient's heart rate, one focuses on the variables (the heart rate) that correspond to the activities (recording heart rate). The resource perspective refers to the actors who perform the activities. Conformance checking from a resource perspective may include comparing resource restrictions with the behaviour seen in the log. Last, the time perspective is relevant in terms of conformance when there are certain time constraints in place. Examples of time aspects that can be analysed from the log are processing time, i.e. the time it takes for an activity to finish, and waiting time, i.e. the time between two activities.

In this study, we respond to the call of exploring the use of qualitative as well as quantitative methods to detect workarounds. Specifically, we focus on the detection of workarounds in healthcare, which has currently most often been studied qualitatively. Process mining techniques have been used in several healthcare case studies before [22], but none of them have focused on the detection of workarounds specifically. We draw on conformance checking techniques, an area of process mining that is relatively underexposed. Additionally, we take a broad perspective on processes, paying equal attention to the data, resource and time perspectives as on the control-flow perspective.

\section{Research Method}

We conducted a multiple-case study, involving six Dutch healthcare organisations (Table 1). In cases A through $\mathrm{E}$ we used qualitative methods to detect 51 workarounds, using observations and interviews. In [3] we report on the detailed methods used in these cases. Below, we focus specifically on the research methods used in case study $\mathrm{F}$, where we used quantitative techniques to detect the workarounds identified in cases A through E. All six case studies were executed in line with the ethical procedures of Utrecht University and the hospitals of study. The involved participants from the hospital have given consent to the researcher to gather data on the workarounds and report on them. As to ensure compliance with the General Data Protection Rights (GDPR) data regulations, no individual data of patients or employees were collected. All data extracted for process mining were anonymised before they were provided to the researcher, through end-toend encrypted servers.

Table 1. Overview of Case Organisations

\begin{tabular}{|l|l|l|}
\hline Case & $\begin{array}{l}\text { Organization } \\
\text { type }\end{array}$ & Department \\
\hline A & General hospital & $\begin{array}{l}\text { Orthopaedics and } \\
\text { surgery }\end{array}$ \\
\hline B & District hospital & Urology and cardiology \\
\hline C & District hospital & Urology and pulmonary \\
\hline D & Specialized centre & Rehabilitation \\
\hline E & Specialized centre & Rehabilitation \\
\hline F & Top clinical & Clinical wards \\
\hline
\end{tabular}

Case study F has taken place at a Dutch top clinical hospital, which admits around forty thousand patients a year. The hospital uses a Health Information System (HIS) that is supplied by one of two main vendors in the Netherlands. Supporting the project, a core team was composed that consisted of a policy officer, a nurse, an IT application manager, a business intelligence specialist, and the first author of this paper. From here on, we will refer to this team as the hospital workarounds team. We will refer to the authors of this paper as the research team. 


\subsection{Data Collection}

In consultation with the hospital workarounds team, we - as research team - chose the clinical care departments as the focus of our study, in particular focusing on nurses. Nurses are especially known for their use of workarounds $[9,13,29]$ and choosing the clinics as the area of focus allows for analysis of the interaction of nurses with other caregivers and professionals, besides patients. This interaction between different healthcare professionals has proven to be an active breeding ground for workarounds [3]. The main processes that involve nurses in clinics include treating, transferring, and discharging clinical patients. To bring further focus to our work, we made the decision to focus on the set of processes that fall under the main process of treating a clinical patient.

Even for the process of treating a clinical patient, a hospital of this size gathers a tremendous amount of process data. To get a good understanding of where workarounds might be found, we chose one document as the base for our analysis: the official hospital handbook that lists all formalised agreements on how caregivers are to work with the HIS. We used this handbook as the description of the intended, normative behaviour.

To determine which processes might contain workarounds, we drew on the list of 51 workarounds identified using observations and interviews with healthcare professionals during case studies A through E. For each workaround in the list, we determined whether it could potentially occur in hospital $\mathrm{F}$ as well, taking into account the scope and specifics of our study. We categorised each of the relevant workarounds into four process perspectives, according to the nature of the deviation. Last, the remaining workarounds were discussed with the hospital workarounds team. For the purpose of this study, the team chose two typical workarounds of each category that were feasible to explore using a data-driven approach. Table 2 illustrates the processes and perspectives the workarounds belong to. For example, in the process of screening a patient for malnutrition, one control-flow workaround, one data workaround, and one resource workaround were identified.

Table 2. Workaround Types Found per Process (Control-flow, Time, Data, and Resource)

\begin{tabular}{|l|l|l|l|l|}
\hline Process & C & T & D & R \\
\hline $\begin{array}{l}\text { Screening a patient for } \\
\text { malnutrition (1) }\end{array}$ & $\mathrm{x}$ & & $\mathrm{x}$ & $\mathrm{x}$ \\
\hline $\begin{array}{l}\text { Recording the vital signs of a } \\
\text { patient (2) }\end{array}$ & $\mathrm{x}$ & $2 \mathrm{x}$ & & \\
\hline Placing a medication order (3) & & & $\mathrm{x}$ & $\mathrm{x}$ \\
\hline
\end{tabular}

The data necessary for analysing the workarounds were pseudonymised and provided to the research team by the business intelligence department of the hospital. We then transformed the data to the required event log format using Power Query. We created four event logs: one for each process, with the medication order process being the exception, as this process was separated into two logs. Table $\mathbf{3}$ provides information on the event logs created.

Table 3. Event Logs

\begin{tabular}{|l|l|l|l|l|}
\hline Process & \#cases & \#events & 1 $^{\text {st }}$ event & last event \\
\hline 1 & 33,613 & 169,384 & $2 / 7 / 18$ & $23 / 7 / 20$ \\
\hline 2 & 4,850 & 86,849 & $31 / 8 / 19$ & $13 / 1 / 20$ \\
\hline 3A & 14,874 & 48,697 & $30 / 3 / 18$ & $2 / 8 / 20$ \\
\hline 3B & 10,639 & 35,301 & $31 / 3 / 18$ & $2 / 8 / 20$ \\
\hline
\end{tabular}

After creating the event logs, we used the PAFnow process mining plugin for Power $\mathrm{BI}^{1}$ to guide the interactive sessions with the workarounds team. PAFnow provides a set of custom process mining visualisations that can be used alongside regular data visualisations, allowing for the creation of dashboards not possible using other tools. The algorithm is closed-source but is comparable to the idea outlined in [16].

\subsection{Data Analysis}

The data analysis was again performed in close collaboration with the hospital workarounds team, during three interactive sessions in which all members participated. The analyses were prepared by the research team using the process mining plugin for Power BI. The aim of the sessions was to arrive at patterns that signify the occurrence of workarounds, to which we will from here on refer to as the workaround signs. The workaround signs are used to describe what characteristics we find in the data that can be used to establish the occurrence of a workaround. The sessions were also used as an opportunity to discuss any implications of these workarounds in terms of security and how to address them to improve the processes and increase security.

\subsection{Evaluation}

When consensus on the workaround signs was achieved with the workarounds team, the results were presented to a user group of clinical nurses. This group consisted of eight representatives of the clinical wards, who get together regularly in a formal

\footnotetext{
${ }^{1}$ https://pafnow.com/en/
} 
user meeting. Next, we distributed an online survey to the eight nurse representatives, asking them for each of the workarounds whether they recognised it (1), what their motivation is for using the workaround (2), and whether they think the HIS or agreements need to be changed (3). Of the eight representatives we approached, six responded. The answers to the open questions were coded with either 'motivation' or 'improvement' and included in the corresponding descriptions in section 4 .

\section{Results}

In this section, we discuss the different perspectives of workarounds that we found in the hospital of study. For each perspective, we describe the workarounds on two levels. We first describe the documented agreement, extracted from the handbook as described in Section 3.1. We do so on a rather abstract level. Second, we provide the workaround sign that signifies whether the workaround has occurred, doing so on the same, high level. Then, we give an example of the high level workaround by describing in-depth one of two specific workarounds found in the hospital. We continue by describing the detection of the example workaround on this more indepth, detailed level. Last, we explain what the motivations are of the nurses to use this particular workaround, and what suggestions were collected on improving the clinical process in question. Note that the workarounds that were not described in-depth, follow the same pattern: i.e. the same documented agreement and workaround sign applies.

\subsection{Control-Flow Workaround}

The two control-flow workarounds we found can be described as activities being re-sequenced in the process in order to improve the flexibility and efficiency of the process.

Documented Agreement. A process instance should execute a set of activities in a particular order.

Workaround Sign. For a process instance, all activities are executed, but a certain activity is carried out earlier than normally planned (i.e. two activities are swapped).

Example. The agreement in the process of screening a patient for malnutrition is as follows: nurses screen a patient for malnutrition after they have been hospitalised. However, such screening activities are sometimes brought forward in the process in order to relieve nurses in the clinic. The specific workaround that we found in the case study can be described as follows: caregivers screen patients for malnutrition before they are formally hospitalised, but after arrival at the hospital.

This workaround is illustrated in Figure 3. As the order of activities is different when comparing the designed path (solid line) to the workaround path (dashed line), this is a control-flow workaround.

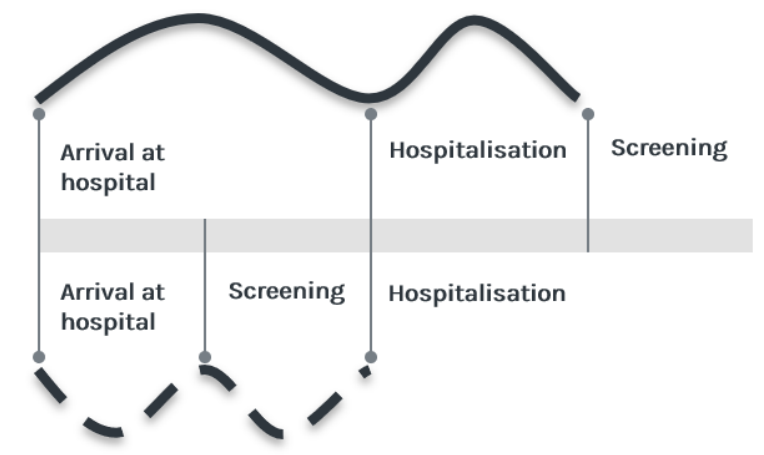

Figure 1. Illustration of Control-Flow Workaround

Detection. In order to detect this workaround, we traced the paths of patients arriving at the hospital. The date and time of the following activities were needed for this purpose: arrival at hospital, hospitalisation, and screening. We determined an instance of a patient arriving at the hospital as a workaround when the following was true: screening was performed after arrival at the hospital, but before hospitalisation. Table 3 provides a snapshot of a process instance extracted from the dataset of the study, that was automatically detected as a workaround.

Table 4. A Detected Control-Flow Workaround

\begin{tabular}{|l|l|l|l|}
\hline $\begin{array}{l}\text { Registration } \\
\text { ID }\end{array}$ & Date & Time & Activity \\
\hline 60933 & $13 / 9 / 2019$ & $15: 39$ & Arrival \\
\hline 60933 & $13 / 9 / 2019$ & $15: 48$ & Screening \\
\hline 60933 & $13 / 9 / 2019$ & $18: 24$ & Hospitalisation \\
\hline
\end{tabular}

Motivation(s) and Improvement. According to the nurses of the clinics, the main motivation for this workaround is to increase efficiency by already performing the screening at the outpatient clinic or during preoperative consultation. As this is a potentially beneficial practice, advancing malnutrition screenings could be encouraged, or even widely institutionalised and supported through the HIS. Shifting tasks to those present at the outpatient clinic or preoperative consultations is likely to leave the nurses at the clinic with more time on their hands with no obvious drawbacks. 


\subsection{Time Workaround}

The two time workarounds we found can be described as activities that are properly executed within the set time constraints, but only reported upon at a later time because of technical or schedule restrictions.

Documented Agreement. A process instance should execute an activity before a certain time or within a certain time constraint.

Workaround Sign. For a process instance, the activity is executed within the time constraint but reported in the system at a later time.

Example. The agreement in the process of recording the vital signs of a patient is as follows: nurses record the vital signs of a patient before the doctor visits (before 9AM, excluding the patients that have been hospitalised on that day). Registering in the system is to be done immediately afterwards. Portable computers are available to support this process. However, a specific workaround that was detected in the case study is that nurses record patient scores within the designated time frame, but only register so after the specified time. They use paper or notebooks to keep track of the scores and sit down behind a computer later in their shifts.

This workaround is illustrated in Figure 4. As the difference between the designed path and the workaround path is the time of registration, this is a time workaround.

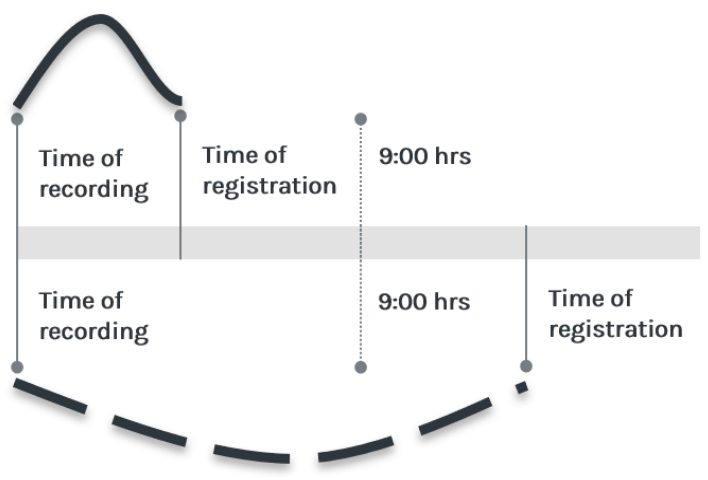

Figure 2. Illustration of Time Workaround

Detection. In order to detect this workaround, we needed to trace the time of recording and time of registration of vital signs of a patient. The registration time is automatically logged by the system and nurses enter the time of recording manually. We determined an instance of a vital signs recording as a workaround when the following was true: time of recording was before $9 A M$, but time of registration was completed after $9 A M$. Table 4 provides a snapshot of a process instance extracted from the dataset of the study, that was automatically detected as a workaround.

Table 5. A Detected Time Workaround

\begin{tabular}{|l|l|l|l|}
\hline Registration ID & Date & Time & Activity \\
\hline 89 & $1 / 10 / 2019$ & $08: 00$ & Recording \\
\hline 89 & $1 / 10 / 2019$ & $11: 04$ & Registration \\
\hline
\end{tabular}

Motivation(s) and Improvement. Nurses note that they experience significant time pressure before visits, such that it is easier to register the recordings later. Also, there is a shortage of portable computers, particularly around 9AM. By registering the recordings on different times during the day, the use of computers is less of a problem. This process can be improved by providing the nurses with more portable computers, or by setting different time constraints in order for the use of portable computers to be more distributed over the day.

\subsection{Data Workaround}

The two data workarounds we found can be described as performing an activity that would not need to be executed according to the value associated with the case because of additional knowledge or other reasons.

Documented Agreement. A process instance should execute an activity when the activity is associated to a certain data value or the data value is within a certain range.

Workaround Sign. For a process instance, the activity is executed even though the value was not equal to the supposed value or not within the supposed range.

Example. The agreement in the process of screening a patient for malnutrition is as follows: the result of the malnutrition screening of a patient is a value from 0 to 7 . When the value is equal to or higher than 3 , nurses need to order a consultation with a dietician. The system supports this decision process, by presenting the user with an advice based on the value and providing them with a shortcut to organise the consultation. However, a specific workaround that was detected in the case study is that of nurses planning a consultation with a dietician, even though the malnutrition value is less than 3 .

This workaround is illustrated in Figure 5. As the difference between the designed path and the workaround path is the value of the malnutrition screening, this is a data-flow workaround. 


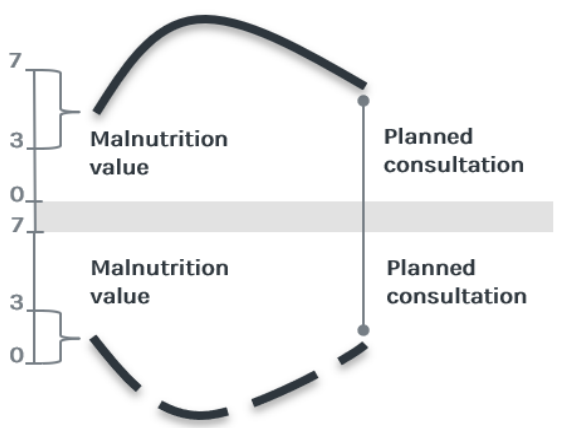

Figure 3. Illustration of Data-Flow Workaround

Detection. In order to detect this workaround, we needed to trace whether a patient's malnutrition screening was followed by a planned consultation. Additionally, we needed to capture the value of the malnutrition screening. We determined an instance of a malnutrition screening of a patient as a workaround when the following was true: malnutrition value was less than 3 and a consultation was planned. Table 5 provides a snapshot of a process instance extracted from the dataset of the study, that was automatically detected as a workaround.

Table 6. A Detected Data Workaround

\begin{tabular}{|l|l|l|l|}
\hline $\begin{array}{l}\text { Registration } \\
\text { ID }\end{array}$ & Date & Activity & Value \\
\hline 37230 & $5 / 1 / 2020$ & Recording & 1 \\
\hline 37230 & $6 / 1 / 2020$ & Consultation & N.a. \\
\hline
\end{tabular}

Motivation(s) and Improvement. According to the nurses of the clinics, there are clinical factors outside the scope of the malnutrition screening that make nurses decide to order a dietician consultation. For example, patients with swallowing problems in need of tube feeding do not necessarily achieve a malnutrition value of 3 or higher, but do benefit from a consultation with a dietician. The process can be improved by including in the advice other important clinical factors besides the malnutrition value.

\subsection{Resource Workaround}

The two resource workarounds we found can be described as resources performing an activity outside of their responsibility, because of abstinence of the responsible actor.

Documented Agreement. An activity should be executed by a specific actor type (e.g. nurse or physician).
Workaround Sign. For a process instance, the activity is executed by a different actor type.

Example. The agreement in the process of placing a medication order is as follows: physicians and specialised nurses prescribe medication for patients, after which they themselves or regular nurses administer the medication. In emergency situations, nurses can employ a one-time medication order to place and sign an order that was not prescribed by the physician or specialised nurse. However, the specific workaround that was detected in the case study was that of nurses using one-time medication orders in non-emergency circumstances.

This workaround is illustrated in Figure 6. As the difference between the designed path and the workaround path is the actor type performing the activity of prescribing medication, this is a resource workaround.

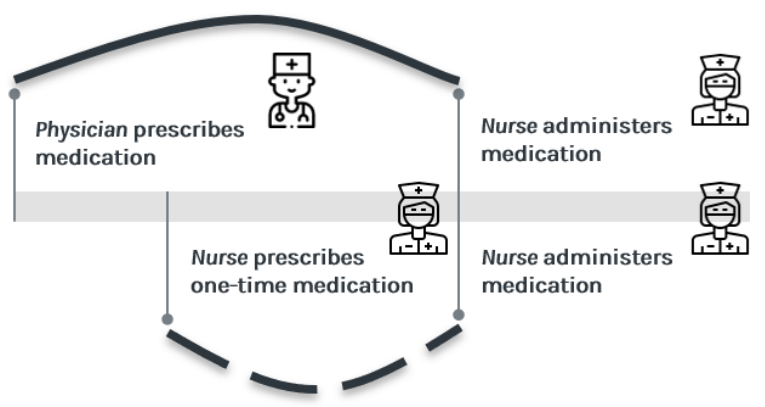

Figure 4. Illustration of Resource Workaround

Detection. In order to detect this workaround, we needed to trace the one-time medication orders used by nurses. However, by merely tracking the one-time medication orders, it does not become clear whether a specific instance has been an emergency situation or not. Therefore, to put these numbers into perspective, we needed to run a comparison to the total number of medication orders of that ward. Thus, we determined an instance of a one-time medication order as a workaround when the following was true: the onetime medication order is beyond the threshold percentage comparing one-time orders to the total number of medication orders of the ward. Table 6 provides a snapshot of a process instance extracted from the dataset of the study, that was automatically detected as a workaround. On the surface, this specific event resembles a normal case of a ward entering a one-time medication order. However, this particular ward frequently orders one-time medication, much more than other wards when comparing total medication orders. 
Table 7. A Detected Resource Workaround

\begin{tabular}{|l|l|l|l|}
\hline Ward ID & Date & Time & Activity \\
\hline 10102033 & $6 / 2 / 2020$ & $16: 42$ & Morphine \\
\hline
\end{tabular}

Motivation(s) and Improvement. According to the consulted nurses, they use the one-time medication order in non-emergency situations when the physician is either unavailable (e.g. at home or in the operating room) or not prepared to enter the prescription. The overall process can be improved by better supporting physicians in the prescription of medication, e.g. by configuring the system in such a way that they are reminded of this and advised that it saves them time to follow up on this advice.

\section{Discussion}

In this study, we performed five qualitative case studies in healthcare organisations to identify 51 workarounds using observations and interviews. In the sixth case study, we detected eight of those using the quantitative method of process mining. The detection and analysis of these workarounds revealed a number of insights related to the different levels of information on which workarounds can be described, their use as a source of organisational improvement, the combination of different process perspectives for improving workaround detection, techniques for detection, and the combination of qualitative and quantitative methods for studying workarounds.

\subsection{Different Levels of Workaround Information}

Workarounds can be described on different levels: a high, very general, level, and a lower, more specific level. On a high level, workarounds in healthcare seem similar to those that take place within other sectors. When comparing our high-level workarounds to the ones identified in relation to purchasing and intake processes [19], some, but not all, are quite similar. For example, in a purchasing process, the general workaround 'Bypassing process parts' was identified, referring to activities that were bypassed such that other activities were performed before their time. Similarities can be found with our control-flow workaround (Section 4.1). Another workaround that was identified in a purchasing process was 'Incompliance to role definition' where resources perform activities not under their responsibility, similar to our resource workaround (Section 4.4).

The differences between workarounds in healthcare and other sectors reside on the more detailed level. On the more detailed level, the title
'Bypassing process parts' does not do justice to the care process workaround that we found. Bypassing activities or skipping them altogether has negative connotations, whereas the workaround we found was anything but negative. Likewise, although the identified resource workaround would fit best in the category 'Incompliance to role definition', it is not the nurse who commits incompliance: the workaround is rather a way of how nurses flexibly respond to the behaviour of physicians.

By generalising workarounds into high-level workaround types, information is lost on the complex interactions between actors and the system, interactions that tell the story of how the workarounds came to be [3]. What is also "lost in translation" is the clinical knowledge of the actors, as well as other contextual information. There is room for further tapping into the potential of the data and time perspective to enrich process analyses with more context, thereby giving broader insights into the environment surrounding workarounds.

\subsection{Workarounds as a Source of Organisational Improvement}

Organisations can respond to workarounds in different ways and choosing the right response depends on the context $[4,5,21]$. According to Boudreau et al. [8], sharing workarounds can be seen as a process of knowledge management. Indeed, our results show that sharing workarounds may benefit the organisation. If deviations such as bringing forward screening activities are formalised across departments, it would leave clinical nurses with more time on their hands. If information on the limited number of portable computers would be shared across the organisation, there may well be solutions available. Demystifying the use of workarounds and antecedents for using them is key in improving the processes in which they occur [20, 23, 24].

Simply checking whether users conform to documented procedures may give an incomplete and possibly harmful picture of work done. For example, hospitals might check the conformance of the malnutrition screening process. They might extract data on the hospitalisation of patients and analyse in how many cases this hospitalisation was followed by a malnutrition screening. However, this would exclude all patients who were already screened before hospitalisation and thus present a number that is too pessimistic. A more comprehensive picture would be gathered by taking into account the workaround of activities being brought forward in the process. Similarly, in the same process of malnutrition screening, a hospital might be interested 
in the conformance to the rule that malnutrition values between 3 and 7 are followed by a consultation with a dietician. Merely analysing the patients having received a malnutrition value in that range would exclude patients who received a lower value but consulted with a dietician anyway. Taking into account the use of workarounds - whether or not detected using qualitative methods - in quantitative analyses will improve data quality and subsequently the quality of process analyses.

\subsection{Improving Workaround Detection by Combining Process Perspectives}

The discussed examples of the four perspectives demonstrate that workarounds can occur in very different shapes and sizes. The multi-perspective approach not only helped categorise the workarounds but can also be used to guide their detection. An interesting avenue for future work is to combine different process perspectives to enable a more precise detection of workarounds. For example, consider the time workaround example (Section 4.2) identified in this study. We checked whether the time of recording was completed within the time constraint and the registration was completed afterwards. Combining this with a resource perspective, we might check whether multiple cases where this behaviour is found are ascribed to the same resource, making it plausible that a particular nurse registered multiple recordings in batch.

\subsection{Process Mining Techniques to Detect Multi-Perspective Workarounds}

In this study, we used PAFnow to detect the workarounds because of the following reasons. First, the hospital uses Power BI, and as PAFnow is a plugin for Power BI, it allows the organisation to integrate the created dashboards into their current tooling and monitor the workarounds over time. Second, the custom process mining visualisations can be used alongside a broad array of other visualisations offered by Power BI, allowing for the creation of dashboards incorporating different process perspectives. It is worth mentioning that the workarounds can also be detected using other process mining techniques and tools, such as Disco and Celonis. One can also model each workaround sign as a data-aware Petri net and use the multiperspective conformance checking technique to detect the workarounds [1, 17].

\subsection{Combining Qualitative and Quantitative Methods for Studying Workarounds}

The final major insight that can be drawn from this study is that different research methods are necessary to detect and understand the use of workarounds in practice, which confirms earlier studies on workarounds. As Ejnefjäll and Ågerfalk stated: "Since workaround behaviors can take different forms in different settings, we need to understand the context and phenomena before using quantitative data-collection methods, which makes studying workarounds ideal for multi-method research that combines qualitative and quantitative methods" [10]. Indeed, in order to detect workarounds, one must first learn what the designed paths are and what that behaviour looks like in the data, before one can start identifying workarounds. However, as mentioned earlier in this discussion, even when there is a documented model of intended behaviour to compare the logged behaviour to, not all workarounds will be detected. We propose the use of a repository of known workarounds that have been identified using qualitative methods. This way, there is a starting point for the quantitative process mining analysis. The process mining analysis in turn can help extend the repository with new workarounds detected. As such, a combination of both qualitative and quantitative methods enables precise and indepth understanding of workarounds and the reasons they exist. Future work may focus on further building this repository of workarounds that exist in different types of organisations and the formation of new and more precise workaround signs that help detect and analyse them. It may also focus on the ways organisations can best respond to them and how workarounds evolve over time. Recent techniques around process drift detection [7] can be relevant instruments for revealing this evolution.

\section{Conclusion}

Whereas workarounds have commonly been studied in healthcare, they have only been identified using labour-intensive qualitative methods that possibly give an incomplete picture. In this study, we identified 51 workarounds using qualitative methods and detected eight of them using the quantitative technique of process mining, each viewed from a different process perspective. We demonstrate how very diverse workarounds can be translated to generic workaround signs, which describe characteristics that can be detected in the data using process mining techniques. Once identified, they can be used for 
process management and organisational improvement. Our work shows the way forward to use quantitative methods in addition to qualitative methods, to detect workarounds in the challenging but highly relevant healthcare environment.

\section{References}

[1] van der Aalst, W., Process Mining: Data Science in Action, Springer, 2016.

[2] Alter, S., "Theory of Workarounds", Communications of the Association for Information Systems 34(55), 2014, pp. 1041-1066.

[3] Beerepoot, I., J.J. Koorn, I. van de Weerd, B. van den Hooff, H. Leopold, and H. Reijers, "Working around health information systems: The role of power", ICIS, (2019).

[4] Beerepoot, I., A. Ouali, I. van de Weerd, and H.A. Reijers, "Working around health information systems: To accept or not to accept?", ECIS, (2019).

[5] Beerepoot, I., and I. van de Weerd, "Prevent, redesign, adopt or ignore: Improving healthcare using knowledge of workarounds", European Conference on Information Systems, (2018).

[6] van Beijsterveld, J.A.A., and W.J.H. van Groenendaal, "Solving misfits in ERP implementations by SMEs", Information Systems Journal 26(4), 2016, pp. 369-393.

[7] Bose, R.P.J.C., W.M.P. van der Aalst, I. Žliobaite, and M. Pechenizkiy, "Handling concept drift in process mining”, Lecture Notes in Computer Science, Springer, Berlin, Heidelberg (2011), 391-405.

[8] Boudreau, P., D. Vieru, G. Paquette, and M. Heon, "The Workarounds Process as a Source of Knowledge Creation and Management", HICSS, (2016), 4292-4301. [9] Debono, D.S., D. Greenfield, J.F. Travaglia, et al., "Nurses' workarounds in acute healthcare settings: a scoping review", BMC health services research 13(1), 2013, pp. 175.

[10] Ejnefjäll, T., and P.J. Ågerfalk, “Conceptualizing Workarounds: Meanings and Manifestations in Information Systems Research", Communications of the Association for Information Systems 45(1), 2019, pp. 20.

[11] Ferneley, E.H., and P. Sobreperez, "Resist, comply or workaround? An examination of different facets of user engagement with information systems", European Journal of Information Systems 15(4), 2006, pp. 345-356.

[12] Halbesleben, J.R.B., G.T. Savage, D.S. Wakefield, and B.J. Wakefield, "Rework and workarounds in nurse medication administration process: implications for work processes and patient safety", Health care management review 35(2), 2010, pp. 124-133.

[13] Koppel, R., S.W. Smith, J. Blythe, and V. Kothari,

"Workarounds to computer access in healthcare organizations: you want my password or a dead patient?", ITCH, (2015), 215-220.

[14] Koppel, R., T. Wetterneck, J.L. Telles, and B.T. Karsh, "Workarounds to Barcode Medication Administration Systems: Their Occurrences, Causes, and
Threats to Patient Safety", Journal of the American Medical Informatics Association 15(4), 2008, pp. 408-423. [15] Laumer, S., C. Maier, and T. Weitzel, "Information quality, user satisfaction, and the manifestation of workarounds: a qualitative and quantitative study of enterprise content management system users", European Journal of Information Systems, 2017, pp. 1-28.

[16] Leemans, S.J.J., E. Poppe, and M.T. Wynn, "Directly follows-based process mining: Exploration \& a case study", ICPM, (2019), 25-32.

[17] Mannhardt, F., M. De Leoni, H.A. Reijers, and W.M.P. Van Der Aalst, "Balanced multi-perspective checking of process conformance", Computing 98(4), 2016, pp. 407-437.

[18] Munoz-Gama, J., Conformance checking and diagnosis in process mining, Springer, 2016.

[19] Outmazgin, N., and P. Soffer, "A process miningbased analysis of business process work-arounds", Software \& Systems Modeling 15(2), 2016, pp. 309-323. [20] Petrides, L.A., S.I. McClelland, and T.R. Nodine, "Costs and benefits of the workaround: Inventive solution or costly alternative", International Journal of Educational Management 18(2), 2004, pp. 100-108.

[21] Röder, N., M. Wiesche, M. Schermann, and H. Krcmar, "Toward an Ontology of Workarounds: A Literature Review on Existing Concepts", HICSS, (2016), 5177-5186.

[22] Rojas, E., J. Munoz-Gama, M. Sepúlveda, and D. Capurro, "Process mining in healthcare: A literature review", Journal of Biomedical Informatics 61, 2016, pp. 224-236.

[23] Safadi, H., and S. Faraj, "The role of workarounds during an opensource electronic medical record system implementation", ICIS, (2010).

[24] Vogelsmeier, A.A., J.R.B. Halbesleben, and J.R. Scott-Cawiezell, "Technology implementation and workarounds in the nursing home", Journal of the American Medical Informatics Association 15(1), 2008, pp. 114-119.

[25] Wager, K.A., F.W. Lee, and J.P. Glaser, Health care information systems: a practical approach for health care management, John Wiley \& Sons, 2017.

[26] van de Weerd, I., P. Vollers, I. Beerepoot, and M. Fantinato, "Workarounds in retail work systems: prevent, redesign, adopt or ignore?", ECIS, 2019.

[27] Weinzierl, S., V. Wolf, T. Pauli, D. Beverungen, and M. Matzner, "Detecting Workarounds in Business Processes-a Deep Learning method for Analyzing Event Logs.", ECIS, (2020).

[28] Winter, A., R. Haux, E. Ammenwerth, B. Brigl, N. Hellrung, and F. Jahn, "Health information systems", In Health Information Systems. Springer, 2010, 33-42. [29] Yang, Z., B.Y. Ng, A. Kankanhalli, and J.W. Luen Yip, "Workarounds in the use of IS in healthcare: A case study of an electronic medication administration system", International Journal of Human Computer Studies 70(1), 2012, pp. 43-65. 